\title{
TMEM88 is a Prognostic Biomarker and Correlates with Immune Infiltrates in Hepatocellular Carcinoma
}

\author{
Andrew Liman \\ Zhongnan Hospital of Wuhan University \\ Yang Gu \\ First People's Hospital of Jingmen \\ PengPeng Liu \\ Zhongnan Hospital of Wuhan University \\ Quanyan Liu ( $\square$ lqy@whu.edu.cn ) \\ Tianjin Medical University General Hospital
}

\section{Research Article}

Keywords: TMEM88, Transmembrane protein 88, HCC, Liver Cancer, Hepatocellular Carcinoma

Posted Date: February 3rd, 2022

DOI: https://doi.org/10.21203/rs.3.rs-1024755/v2

License: (a) (i) This work is licensed under a Creative Commons Attribution 4.0 International License. Read Full License 


\section{Abstract}

Background: Transmembrane protein 88 (TMEM88) has emerged as a newly discovered cancer-related protein that acts as a cancer-promoting or cancer-inhibiting regulator in multiple tumor types. However, the exact role of TMEM88 in liver cancer is undetermined. The current study was designed to determine the expression of TMEM88 in liver cancer.

Methods: We used data provided by public databases TCGA and GEO, analyzed them with Kaplan-Meier, TIMER and GEPIA.

Results: TMEM88 expression was significantly lower in several human cancers, but higher in liver and bile cancer, than in corresponding normal tissues. TMEM88 expression in HCC tissues correlated with prognosis. Low TMEM88 expression associated with poorer overall survival, disease-specific survival, progression-free survival, and relapse-free survival in multiple cohorts of HCC patients, particularly at late disease stages (grade 2 and 3 ). TMEM88 showed strong correlation with tumor-infiltrating B cells, CD4+ and CD8+ T cells, macrophages, neutrophils, and dendritic cells.

Conclusion: These findings demonstrate that TMEM88 is a potential prognostic biomarker that determines cancer progression and correlated with tumor immune cells infiltration in HCC.

\section{Introduction}

Hepatocellular carcinoma (HCC) is among the most lethal and prevalent cancers in the human population, which is more common in men than in women. ${ }^{1}$ It accounts for at least $85 \%$ of primary liver cancer which is the third most common cause of cancer mortality worldwide compared with secondary liver cancer. ${ }^{2}$ The global incidence of HCC shows dramatical differences on its worldwide distribution, gender, race, and region. The risk factors for HCC then vary by many aspects, making the patients with HCC having a highly multiple courses. ${ }^{3,4}$

Survival has improved due to the advancement of the diagnostic time (lead-time bias), and the effective therapeutic strategies with the current 5 -year survival in surgical patients might had exceeded $70 \% .5,6$ notion is supported that HCC comprises several biologically distinct subgroups with molecular heterogeneity that has not been appreciated from methods traditionally used to characterize HCC. Cancer staging therefore becomes a critical step in management of HCC. However, although improving the classification of HCC patients would at least improve the application of currently available treatment modalities for better prognosis, the prognosis of HCC is complex and multifaceted still. ${ }^{7}$ The optimal staging system is still under intense debate despite that key predictors of prognosis are recognized as tumor extent, degree of liver dysfunction, and patient's general health condition. ${ }^{7,8}$ But it is as true as with other cancers, the early diagnosis and early treatment of HCC can significantly improve the overall effect. Recent researches have confirmed that the occurrence of liver cancer is closely related with many oncogenes and tumor suppressor genes and that these genes' expressions and their

product structures at the cell or molecular level would contribute to explore the carcinogenesis of HCC. ${ }^{9-11}$ Besides, studying genes from genetic level for diagnosis, treatment, and prevention of HCC to explore cancerrelated genes and protein as detecting molecular biomarkers opens up a new way for the study on the 
pathogenesis of HCC as well. Recently, many biomarkers were identified relating to invasion, metastasis, recurrence, and survival, such as transcripts of tumor-associated antigens (a-fetoprotein (AFP), melanomaassociated antigens (MAGEs), and cytokeratin 19 (CK19)), ${ }^{12,13}$ the molecular markers for cellular malignancy phenotype (DNA ploidy, ${ }^{14}$ cellular proliferation index, ${ }^{15,16}$ cell cycle regulators, ${ }^{17}$ oncogenes, and tumor suppressors ${ }^{18,19}$ ), as well as telomerase activity. ${ }^{20}$ Some of these biomarkers are potential predictors for HCC metastatic recurrence and clinical outcomes. ${ }^{21}$ Accordingly, the identification and clinical application of the prognostic biomarkers for $\mathrm{HCC}$ are feasible and promising.

Transmembrane protein 88 (TMEM88) is a newly discovered transmembrane protein that interacts with dishevelled-1 protein during embryonic development. ${ }^{22}$ It has been well documented that TMEM 88 functions as a key regulator of $\mathrm{Wnt} / \beta$-catenin signaling and plays a pivotal role in regulating cardiomyocyte differentiation and heart development in embryos. ${ }^{23}$ The dysregulation of TMEM88 is implicated in multiple pathological processes, including keloid formation, inflammation, and liver fibrosis. ${ }^{24-26}$ Notably, TMEM88 has emerged as a key regulator in cancer initiation and progression; it functions as an oncogene or a tumorsuppressor. ${ }^{27}$ TMEM88 expression is frequently elevated in lung and breast cancers, a phenomenon that is correlated with tumor advance, lymph node metastasis, and a poor overall survival rate. ${ }^{28,29}$ Moreover, TMEM88 overexpression enhances the invasion and metastasis of cancer cells in vitro and in vivo. ${ }^{28,29}$ In contrast, low TMEM88 expression is found in non-small cell lung cancer due to hypomethylation of its promoter, and its overexpression restricts cancer cell proliferation and invasion. ${ }^{30}$ These findings suggest an essential role for TMEM88 in cancer progression.

Unfortunately, the expression pattern of this protein in normal liver cells and liver tumor cells has not been investigated, let alone the association of this gene and the prognosis of HCC patients. Accordingly, TMEM88 was newly screened out as prognostic marker of HCC in this study.

\section{Methods And Materials}

The source of all data in this research are belong to public databases TCGA and GEO. Users can download relevant data for free for research and publish relevant articles. Our study is based on open source data, so there are no ethical issues and other conflicts of interest.

The Cancer Genome Atlas (TCGA), a landmark cancer genomics program, molecularly characterized over 20,000 primary cancer and matched normal samples spanning 33 cancer types. This joint effort between NC and the National Human Genome Research Institute began in 2006, bringing together researchers from diverse disciplines and multiple institutions.

Over the next dozen years, TCGA generated over 2.5 petabytes of genomic, epigenomic, transcriptomic, and proteomic data. The data, which has already led to improvements in our ability to diagnose, treat, and prevent cancer, will remain publicly available for anyone in the research community to use.『cancer.gov/aboutnci/organization/ccg/research/structural-genomics/tcga》

\section{Kaplan-Meier survival curve analysis}


Kaplan-Meier survival curve analysis was performed to assess the correlation between the expression of the 54,000 genes (mRNA, miRNA, protein) on the survival rates in 21 different cancers using more than 10,000 cancer samples, including 364 liver, 7830 breast, 1440 gastric, 2190 ovarian, and 3452 lung cancer samples. Kaplan-Meier plots (http://kmplot.com/analysis/) were used to analyze the relationship between TMEM88 gene expression and survival rates in liver, gastric, breast, pancreatic, ovarian, and lung cancers based on the hazard ratios (HR) and log-rank P-values. ${ }^{32}$

\section{TIMER analysis}

TIMER database was used to systematically analyze the tumor-infiltrating immune cells (TIICs) in 32 cancer types using more than 10,000 samples from The Cancer Genome Atlas

(TCGA) (https://cistrome.shinyapps.io/timer/) database. ${ }^{31}$ TIMER determines the abundance of tumorinfiltrating immune cells (TIICs) based on the statistical analysis of gene expression profiles. ${ }^{33}$ We analyzed the association between the level of TMEM88 gene expression and the abundance of infiltrating immune cells, including CD4+ T cells, CD8+ T cells, B cells, neutrophils, dendritic cells and macrophages based on expression of specific marker genes in different cancers including HCC. The marker genes used for analysis of tumorinfiltrating immune cells including T cells, B cells, TAMs, monocytes, M1 macrophages, M2 macrophages, natural killer (NK) cells, neutrophils, dendritic cells (DCs), T-helper (Th) cells, T-helper 17 (Th17) cells, follicular

helper T (Tfh) cells, exhausted T cells, and Tregs were based on data from previous studies. ${ }^{34,35}$ TMEM88 gene was on the $\mathrm{x}$-axis and related marker genes are on the $\mathrm{y}$-axis.

\section{GEPIA analysis}

The Gene Expression Profiling Interactive Analysis (GEPIA) database (http://gepia.cancer-pku.cn/index. html) was used to analyze the RNA sequencing expression data from 8,587 normal and 9,736 tumor tissue samples from the TCGA and GTEx projects. ${ }^{36}$

\section{Results}

\section{The levels of TMEM88 mRNA in HCC and other cancers}

The analysis of TCGA RNA-seq data using the TIMER database showed that TMEM88 mRNA expression was significantly lower in BLCA (bladder urothelial carcinoma), BRCA (breast invasive carcinoma), CESC (Cervical squamous cell carcinoma and endocervical adenocarccinoma), KICH (kidney chromophobe), KIRP (kidney renal papillary carcinoma), LUAD (lung adenocarcinoma), LUSC (lung squamous cell carcinoma), READ (rectum adenocarcinoma), THYM (Thymoma), and UCEC (uterine corpus endometrial carcinoma) tissues compared with the corresponding normal tissues (Figure 1). TMEM88 mRNA expression was higher in HCC (Liver hepatocellular carcinoma) and CHOL (Cholangiocarcinoma) compared with the corresponding normal tissues. These data showed that TMEM88 expression was deregulated in most of the cancer tissues but in the liver cancer tissue.

\section{Prognostic significance of TMEM88 expression in human cancers}


Next, we analyzed the prognostic value of TMEM88 expression in human cancers using the Kaplan-Meier plotter database. Low TMEM88 expression was associated with poorer prognosis in HCC (OS: HR $=0.58,95 \%$ $\mathrm{Cl}=0.41$ to 0.82 , $\mathrm{P}=0.0018$; $\mathrm{PFS}$ : $\mathrm{HR}=0.59,95 \% \mathrm{Cl}=0.44$ to $0.8, \mathrm{P}=0.00047$; RFS: $\mathrm{HR}=0.69,95 \% \mathrm{Cl}=0.49$ to 0.96 , $P=0.026$; DSS: $H R=0.49,95 \% \mathrm{Cl}=0.31$ to $0.78, P=0.0018$; Figure $2 A-2 D$ ), Breast Cancer (RFS: $H R=0.67,95 \%$ $\mathrm{Cl}=0.58$ to $0.78, \mathrm{P}=2.4 \mathrm{e}-07$; Figure $2 \mathrm{G}$ ) and Pancreatic Ductal Adenocarcinoma (OS: $\mathrm{HR}=0.68,95 \% \mathrm{Cl}=0.45$ to $1.03, \mathrm{P}=0.066$; RFS: $\mathrm{HR}=0.5,95 \% \mathrm{Cl}=0.21$ to $1.19, \mathrm{P}=0.11$; Figure $2 \mathrm{~L}, 2 \mathrm{M})$. Low TMEM88 expression was associated with better prognosis in Breast Cancer (PPS: $\mathrm{HR}=1.37,95 \% \mathrm{CL}=0.96$ to 1.94, $\mathrm{P}=0.083$; Figure 2F), Gastric Cancer (OS: $\mathrm{HR}=1.74,95 \% \mathrm{Cl}=1.4$ to 2.17, $\mathrm{P}=4.2 \mathrm{e}-07$; PPS: $\mathrm{HR}=2.35,95 \% \mathrm{Cl}=1.77$ to $3.13, \mathrm{P}=1.3 \mathrm{e}-0$; FP: $\mathrm{HR}=1.57,95 \% \mathrm{Cl}=1.23$ to $1.99, \mathrm{P}=0.00023$; Figure $2 \mathrm{I}-2 \mathrm{~K}$ ), Lung Cancer (PPS: $\mathrm{HR}=1.59,95 \% \mathrm{Cl}=1.03$ to 2.46 , $\mathrm{P}=0.034$; Figure 20). However, TMEM88 expression was not associated with OS and DMFS in breast cancer (Figure 2E, 2H), OS and FP in Lung Cancer (Figure 2N, 2P), and OS, PFS, and PPS in Ovarian cancer (Figure $2 \mathrm{Q}-2 \mathrm{~S})$. These results demonstrate the prognostic significance of TMEM88 expression in liver, breast, gastric, pancreatic ductal, and lung cancers.

However TMEM88 mRNA expression was higher in HCC (Liver hepatocellular carcinoma) and CHOL (Cholangiocarcinoma) compared with the corresponding normal tissues but high TMEM88 expression was associated with better prognosis in both HCC and CHOL.(Figure 1 and Figure 2A-2D)

\section{Correlation between TMEM88 expression and infiltration levels of immune cells of HCC patients}

We used $371 \mathrm{HCC}$ patients data in TIMER database to investigate the correlation of TMEM88 expression and infiltration levels of immune cells in HCC tissues (Figure 3). TMEM88 expression in HCC tissues positively correlates with infiltration levels of $\mathrm{CD} 8+\mathrm{T}$ cells (partial.cor $=0.004$ ), but negatively correlates with tumor purity (cor $=-0.178$ ) and infiltration levels of $B$ cells (partial.cor $=-0.207$ ), CD4+ T cells (partial.cor $=-0.065$ ), macrophages (partial.cor=-0.2), neutrophils (partial.cor=-0.145) and dendritic cells (partial.cor=-0.05).

\section{Correlation analysis of TMEM88 and other marker genes of immune cells expression with patients survival in $\mathrm{HCC}$ and $\mathrm{CHOL}$ tissues}

In this investigation we found out that the expression of TMEM88 $(p=0.004)$ in HCC tissues was the only factor that statistically significant correlation with patients survival outcome, the higher TMEM88 expression in HCC patients the better prognosis (Figure 4). The expression of $B$ cells $(p=0.489), C D 8+$ cells $(p=0.551), C D 4+$ cells $(p=0.454)$, macrophages $(p=0.153)$, neutrophils $(p=0.2)$ and dendritic cells $(p=0.288)$ was not have statistically significant correlation with patients survival outcome. On the other hand, the only marker genes have statistically significant correlation with patients survival outcome in $\mathrm{CHOL}$ tissues was in neutrophils $(p=0.044)$, but not in TMEM88 ( $p=0.534), B$ cells $(p=0.619), C D 8+$ cells $(p=0.208), C D 4+$ cells $(p=0.244)$, macrophages $(p=0.124)$, and dendritic cells $(p=0.131)$.

Table 1. Correlation of TMEM88 mRNA expression and prognosis in hepatocellular carcinoma with different clinicopathological factors by Kaplan-Meier plotter. 
Clinicopathological factors survival Overall

N Hazard ratio value
P- N Hazard ratio

survival

Progression-free

P-value

SEX

\begin{tabular}{lllllll} 
Female & 118 & $0.44(0.24-0.79)$ & $\mathbf{0 . 0 0 5}$ & 120 & $0.63(0.38-1.05)$ & 0.0715 \\
\hline Male & 246 & $0.58(0.37-0.91)$ & $\mathbf{0 . 0 1 6}$ & 246 & $0.65(0.45-0.93)$ & $\mathbf{0 . 0 1 7 3}$ \\
\hline Stage & & & & & & \\
\hline 1 & 170 & $0.77(0.42-1.41)$ & 0.3954 & 170 & $0.58(0.35-0.96)$ & 0.0313 \\
\hline 2 & 83 & $0.59(0.27-1.31)$ & 0.1886 & 84 & $0.65(0.36-1.18)$ & 0.1561 \\
\hline $1+2$ & 253 & $0.72(0.45-1.16)$ & 0.1788 & 254 & $0.57(0.39-0.84)$ & 0.0036 \\
\hline 3 & 83 & $0.44(0.24-0.81)$ & $\mathbf{0 . 0 0 6 4}$ & 83 & $0.9(0.53-1.55)$ & 0.7122 \\
\hline $2+3$ & 166 & $0.49(0.3-0.8)$ & $\mathbf{0 . 0 0 3 2}$ & 167 & $0.72(0.48-1.07)$ & 0.1024 \\
\hline 4 & 4 & - & - & 5 & - & - \\
\hline $3+4$ & 87 & $0.5(0.28-0.89)$ & $\mathbf{0 . 0 1 5 8}$ & 88 & $0.81(0.48-1.37)$ & 0.4313 \\
\hline Grade & & & & & & \\
\hline 1 & 55 & $0.59(0.23-1.52)$ & 0.2734 & 55 & $0.72(0.33-1.57)$ & 0.4078 \\
\hline 2 & 174 & $0.53(0.32-0.89)$ & $\mathbf{0 . 0 1 5}$ & 175 & $0.61(0.39-0.94)$ & $\mathbf{0 . 0 2 3 4}$ \\
\hline 3 & 118 & $0.39(0.21-0.75)$ & $\mathbf{0 . 0 0 3}$ & 119 & $0.55(0.33-0.91)$ & $\mathbf{0 . 0 1 7 2}$ \\
\hline 4 & 12 & - & - & 12 & - & - \\
\hline$A J C T$ & & & & & & \\
\hline
\end{tabular}

AJCC_T

\begin{tabular}{lllllll}
1 & 180 & $0.79(0.44-1.41)$ & 0.4186 & 180 & $0.62(0.38-1.02)$ & 0.0557 \\
\hline 2 & 90 & $0.47(0.22-1)$ & $\mathbf{0 . 0 4 6 6}$ & 92 & $0.62(0.36-1.08)$ & 0.0887 \\
3 & 78 & $0.47(0.25-0.86)$ & $\mathbf{0 . 0 1 2 3}$ & 78 & $0.75(0.43-1.33)$ & 0.3288 \\
\hline 4 & 13 & - & - & 13 & - & - \\
\hline Vascular invasion & & & & & & \\
\hline Micro & 90 & $0.46(0.21-1)$ & $\mathbf{0 . 0 4 5 2}$ & 91 & $0.68(0.39-1.2)$ & 0.1818 \\
\hline None & 203 & $0.71(0.43-1.19)$ & 0.194 & 204 & $0.68(0.44-1.06)$ & 0.0888 \\
\hline Race & & & & & & \\
\hline White & 181 & $0.68(0.43-1.07)$ & 0.0967 & 183 & $0.65(0.44-0.97)$ & $\mathbf{0 . 0 3 2 5}$ \\
\hline Asian & 155 & $0.43(0.23-0.8)$ & $\mathbf{0 . 0 0 5 9}$ & 155 & $0.6(0.38-0.97)$ & $\mathbf{0 . 0 3 4 6}$
\end{tabular}

Alcohol consumption 


\begin{tabular}{lcccccc}
\hline yes & 115 & $0.64(0.34-1.22)$ & 0.1684 & 115 & $0.6(0.35-1)$ & $\mathbf{0 . 0 0 8 8}$ \\
\hline none & 202 & $0.5(0.31-0.81)$ & $\mathbf{0 . 0 0 3 7}$ & 204 & $0.66(0.44-0.99)$ & $\mathbf{0 . 0 4 5 6}$ \\
\hline Virus hepatitis & & & & & & \\
\hline Yes & 150 & $1.12(0.58-2.16)$ & 0.7337 & 152 & $0.63(0.39-1)$ & $\mathbf{0 . 0 4 5 9}$ \\
\hline None & 167 & $0.39(0.24-0.63)$ & $\begin{array}{l}7.5 e- \\
05\end{array}$ & 167 & $0.63(0.41-0.98)$ & $\mathbf{0 . 0 3 6 9}$
\end{tabular}

Bold values indicate $\mathrm{P}<0.05$.

\section{Correlation between TMEM88 expression and clinical characteristics of HCC patients}

Next, we investigated the relationship between TMEM88 expression and different clinical characteristics of HCC using the Kaplan-Meier Plotter database and the results are shown in Table 1. Low TMEM88 expression correlated with both poorer OS and PFS in males (OS: HR=0.58, $P=0.0016$; PFS: $\mathrm{HR}=0.65, \mathrm{P}=0.0173$ ), Grade 2 patients (OS: $H R=0.53, P=0.015$; PFS: $H R=0.61, P=0.0234$ ), Grade 3 patients (OS: $H R=0.39, P=0.003$; $P F S: H R=0.55, P=0.0172$ ), Asians (OS: $H R=0.43, P=0.0059 ; P F S: H R=0.6, P=0.0346$ ), and non-alcoholics (OS: $H R=0.5, P=0.0037 ; P F S: H R=0.66, P=0.0456)$. Low TMEM88 mRNA expression correlates with worse OS in female ( $H R=0.44, P=0.005)$, stage $2+3$ patients ( $H R=0.49, P=0.0032)$, stage 3 patients ( $H R=0.44, P=0.0064)$, stage $3+4$ patients $(H R=0.5, P=0.0158)$, AJCC_T grade 2 patients ( $H R=0.47, P=0.0466)$, AJCC_T grade 3 patients $(H R=0.47, P=0.0123)$ and patients with micro vascular invasion $(H R=0.46, P=0.0452)$. Low TMEM88 mRNA expression correlates with worse PFS in stage 1 patients $(H R=0.58, P=0.0313)$, stage $1+2$ patients $(H R=0.57, P=0.0036)$, Whites $(H R=0.65, P=0.0325)$, alcohol consumers $(H R=0.6, P=0.0088)$, patients with hepatitis virus $(H R=0.63, P=0.0459)$ and patients without hepatitis virus $(H R=0.63, P=0.0369)$. However, TMEM88 expression did not correlate with OS and PFS in stage 2 patients (OS: HR=0.59, $P=0.1886$; PFS: $H R=0.65, P=0.1561$ ), grade 1 patients (OS: $H R=0.59, P=0.2734$; $P F S: H R=0.72, P=0.4078$ ) and AJCC_T grade 1 patients (OS: $\mathrm{HR}=0.79, \mathrm{P}=0.4186$; $\mathrm{PFS}$ : $\mathrm{HR}=0.62, \mathrm{P}=0.0557$ ). These results demonstrate that prognostic significance of TMEM88 expression in HCC patients based on their clinical characteristics, especially in late stage of HCC.

Table 2. Correlation analysis between TMEM88 and marker genes of immune cells in GEPIA. 
HCC

CHOL

\begin{tabular}{|c|c|c|c|c|c|c|c|c|c|}
\hline \multirow[t]{2}{*}{ Description } & \multirow[t]{2}{*}{$\begin{array}{l}\text { Gene } \\
\text { markers }\end{array}$} & \multicolumn{2}{|l|}{ Tumor } & \multicolumn{2}{|c|}{ Normal } & \multicolumn{2}{|l|}{ Tumor } & \multicolumn{2}{|c|}{ Normal } \\
\hline & & $\mathbf{R}$ & $P$ & $\mathbf{R}$ & $P$ & $\mathbf{R}$ & $P$ & $\mathbf{R}$ & $\mathbf{P}$ \\
\hline Monocyte & CD86 & -0.1 & 0.054 & 0.57 & $1.8 \mathrm{e}-05$ & 0.05 & 0.77 & 0.87 & 0.0045 \\
\hline \multirow[t]{2}{*}{ Neutrophils } & CD11b & -0.23 & $9.4 \mathrm{e}-06$ & 0.43 & 0.0019 & -0.11 & 0.53 & 0.63 & 0.076 \\
\hline & CCR7 & 0.15 & 0.0035 & 0.49 & 0.00027 & 0.21 & 0.21 & 0.89 & 0.0014 \\
\hline \multirow[t]{2}{*}{ TAM } & CD68 & -0.1 & 0.051 & 0.48 & 0.00039 & -0.24 & 0.15 & 0.75 & 0.025 \\
\hline & IL-10 & -0.032 & 0.54 & 0.2 & 0.17 & 0.018 & 0.92 & -0.18 & 0.65 \\
\hline \multirow[t]{4}{*}{ Th1 } & $\begin{array}{l}\text { IFN-y } \\
\text { (IFNG) }\end{array}$ & -0.14 & 0.0064 & 0.33 & 0.02 & 0.26 & 0.13 & 0.51 & 0.16 \\
\hline & STAT1 & -0.17 & 0.0012 & 0.45 & 0.00091 & -0.24 & 0.15 & 0.3 & 0.44 \\
\hline & $\begin{array}{l}\text { T-bet } \\
\text { (TBX21) }\end{array}$ & 0.17 & 0.00087 & 0.48 & 0.00046 & 0.33 & 0.048 & 0.82 & 0.011 \\
\hline & $\begin{array}{l}\text { TNF-a } \\
\text { (TNF) }\end{array}$ & -0.11 & 0.031 & 0.3 & 0.034 & 0.21 & 0.21 & 0.61 & 0.079 \\
\hline Th2 & STAT6 & 0.059 & 0.26 & 0.48 & 0.00047 & -0.031 & 0.86 & 0.77 & 0.021 \\
\hline \multirow[t]{3}{*}{ Treg } & CCR8 & -0.12 & 0.018 & 0.13 & 0.18 & 0.28 & 0.047 & 0.33 & 0.39 \\
\hline & STAT5B & 0.012 & 0.81 & 0.26 & 0.18 & 0.29 & 0.41 & 0.63 & 0.076 \\
\hline & $\begin{array}{l}\text { TGF- } \beta \\
\text { (TGFB1) }\end{array}$ & -0.062 & 0.23 & 0.7 & $1.9 \mathrm{e}-08$ & 0.06 & 0.73 & 0.83 & 0.0083 \\
\hline \multirow{3}{*}{$\begin{array}{l}\text { T cell } \\
\text { exhaustion }\end{array}$} & CTLA4 & -0.2 & $9.1 \mathrm{e}-05$ & 0.43 & 0.0016 & 0.25 & 0.13 & 0.57 & 0.11 \\
\hline & $\begin{array}{l}\text { PD-1 } \\
\text { (PDCD1) }\end{array}$ & -0.13 & 0.016 & 0.46 & 0.00082 & -0.046 & 0.79 & 0.96 & $4.8 \mathrm{e}-05$ \\
\hline & $\begin{array}{l}\text { TIM-3 } \\
\text { (HAVCR2) }\end{array}$ & -0.18 & $4 \mathrm{e}-04$ & 0.57 & $1.8 \mathrm{e}-05$ & -0.12 & 0.5 & 0.82 & 0.011 \\
\hline
\end{tabular}

Bold values indicate $\mathrm{P}<0.05$.

Correlation analysis between mRNA levels of TMEM88 and markers of different subsets of immune cells

Next, we investigated the correlation between TMEM88 expression and the status of tumor-infiltrating immune cells based on the levels of immune marker gene expression in $\mathrm{HCC}$ and $\mathrm{CHOL}$ tissues using the GEPIA database. The immune cells analyzed in HCC tissues included monocytes, neutrophils, tumor-associated macrophages (TAMs), T helper 1 (Th1), T helper 2 (Th2), regulatory T (Tregs) and exhausted T cells.

Analysis of the GEPIA database showed that TMEM88 expression in HCC tissues significantly correlated with the expression of marker genes from TAMs, neutrophils, T-helper, Treg and exhausted T cells, whereas the 
correlation was not significant in CHOL (Table 2).

Specifically, TMEM88 expression showed significant correlation with the expression of markers of specific immune cells such as monocyte markers, CD86 (CHOL: normal: $\mathrm{R}=0.87, \mathrm{P}=0.0045)$ and CD11b (HCC: normal: $r=0.43, P=0.0019$ ), neutrophils marker, CCR7 (HCC: tumor: $r=0.15, P=0.0035$, normal: $r=0.49, P=0.00027 ; C H O L$ : normal: $r=0.89, P=0.0014$ ), TAM marker, CD68 (HCC: normal: $r=0.48, P=0.00039 ; C H O L$ : normal: $r=0.75$, $P=0.025)$, Th1 markers, IFN-y (HCC: tumor: $r=-0.14, P=0.0064$, normal: $r=0.33, P=0.02)$, STAT1 (HCC: tumor: $r=-0.17, P=0.0012$, normal: $r=0.45, P=0.00091$ ), T-bet (HCC: tumor: $r=0.17, P=0.00087$, normal: $r=0.48$, $\mathrm{P}=0.00046$; CHOL: tumor: $r=0.33, \mathrm{P}=0.048$, normal: $r=0.82, \mathrm{P}=0.011)$, TNF-a (HCC: tumor: $r=-0.11, P=0.031$, normal: $r=0.3, P=0.034$ ), Th2 marker, STAT6 (HCC: normal: $r=0.48, P=0.00047$; $C H O L:$ normal: $r=0.77, P=0.021$ ), Treg marker, CCR8 (HCC: tumor: $r=-0.12, P=0.018$; $C H O L$ : tumor: $r=0.28, P=0.047$ ) and TGF- $\beta$ (CHOL: normal: $r=0.83, P=0.0083$ ), and exhausted T cell, CTLA4 (HCC: normal: $r=0.43, P=0.0016), P D-1$ (HCC: tumor: $r=-0.13$, $\mathrm{P}=0.016$, normal: $r=0.46, \mathrm{P}=0.00082$ ) and TIM-3 (CHOL: normal: $r=0.82, P=0.011)$. These findings strongly suggest that TMEM88 expression correlates with infiltration of immune cells in hepatocellular carcinoma.

\section{Discussion}

The level of TMEM88 mRNA in cancer tissues were analyzed using TIMER, Kaplan-Meier Plotter and GEPIA databases. Analysis of TMEM88 mRNA levels in cancer and normal tissues in the TIMER database revealed that TMEM88 expression was significantly downregulated in most cancer but HCC and CHOL. However, there was variability in the expression of TMEM88 in different types of cancers, which may reflect differences in the data collection methods and the underlying causative mechanism. However, the TMEM88 ecpression data was consistent in HCC tissues across different databases. Gene expression analysis of the GEPIA database revealed that low TMEM88 expression correlated with worse prognosis in the HCC. Kaplan-Meier Plotter analyses showed that low TMEM88 expression correlated with worse prognosis in liver, breast and pancreatic ductal cancers. In HCC patients, low TMEM88 expression correlated with worse prognosis of patients in late stages (grade 2 or grade 3). High TMEM88 expression correlated with better OS, PFS, RFS and DSS in HCC patients. These data strongly suggest that TMEM88 is a potential prognosis biomarker in HCC, especially for patients in late cancer stages.

Immune responses at primary and secondary tumor sites depend on the different types of immune cells that infiltrate into the tumor micro-environment. The infiltration of different types of immune cells is tightly regulated by the various chemokines, which modulate tumor immunity and the biological phenotype of the tumors, and also influence tumor progression, therapy and prognosis ${ }^{33-35}$.

Several factors could influence the outcomes of this study. Firstly, this study is based on data retrieved from published articles, public repositories, and communications with study authors. Hence, the quality of data can influence the study outcomes. Secondly, the quantity of samples in the databases is constantly supervised and extended, which can affect the outcomes of this study. Thirdly, the accuracy and choice of the statistical methods used by the databases to analyze the

data could affect the interpretation of the study results. However, we obtained similar results by analyzing multiple databases, which supports the conclusions of our study. 
Our study has some limitations. Firstly, our investigations into the role of TMEM88 in tumors were based on data that was already reported in the Kaplan-Meier plotter, GEPIA and TIMER databases. However, we did not verify these outcomes by testing our own clinical samples. Secondly, the sample sizes of some individual tumors in the databases were small. In such cases, large sample sizes will be necessary for reliable interpretation of data. Thirdly, we did not conduct animal experiments to confirm the role of TMEM88 in the growth and progression of HCC, and its relationship with the infiltration of immune cells into the tumor microenvironment. Hence, further studies are necessary

to verify the role played by TMEM88 in HCC.

In summary, our results suggest that TMEM88 is a potential independent prognostic biomarker for HCC that can be used to evaluate the levels of immune cell infiltration in the tumor tissues. Relatively high levels of TMEM88 in HCC may indicate greater risk of tumor relapse after treatment and close medical supervision will be necessary for such patients

\section{Conclusion}

This study demonstrate that TMEM88 mRNA levels correlate with prognosis of hepatocellular carcinoma. High TMEM88 mRNA levels correlate with a better prognosis in HCC even though TMEM88 expression in liver cancer cells higher than normal liver cells. The downregulation of TMEM88 associated with worse prognosis in patients associated with clinical characteristics such as males, Asians, non alcohol consumers, Grade 2 and Grade 3 patients. Furthermore, TMEM88 mRNA levels correlate with the numbers of tumor-infiltrated immune cells based on the levels of markers for different immune cell types in HCC. Our study suggest that TMEM88 is a potential prognostic biomarker for HCC.

\section{Abbreviations}

TMEM88: Transmembrane protein 88; HCC: Hepatocellular Carcinoma; TIMER: Tumor Immune Estimation Resource; GEPIA: Gene Expression Profiling Interactive Analysis; OS: overall survival; DSS: disease-specific survival; PFS: progression-free survival; DMFS: distant metastasis-free survival; PPS: post progression survival; FP: first progression.

\section{Declarations}

Consent for publication: Not applicable.

Availability of data and materials: All data generated or analysed during this study are included in this published article.

Competing interests: The authors declare that they have no competing interests.

Funding: Not applicable.

Ethics approval and consent to participate $\$ Not applicable 
Author's contribution: Andrew Liman wrote the main manuscript text, prepared table 1-2 and figures 1-4. Pengpeng Liu and Yang Gu provides research input. All authors reviewed the manuscript. No fund received in this research.

\section{Acknowledgment}

This manuscript has been released as a pre-print at https://doi.org/10.21203/rs.3.rs-1024755/v1. ${ }^{37}$

\section{References}

1. Farazi, PA, Depinho, RA. Hepatocellular carcinoma pathogenesis: from genes to environment. Nat Rev Cancer 2006; 6: 674-687.

2. Jemal, A, Bray, F, Center, MM. Global cancer statistics. CA Cancer J Clin 2011; 61: 33-64.31.

3. Bruix, J, Boix, L, Sala, M. Focus on hepatocellular carcinoma. Cancer Cell 2004; 5: 215-219.

4. Llovet, JM, Bustamante, J, Castells, A. Natural history of untreated nonsurgical hepatocellular carcinoma: rationale for the design and evaluation of therapeutic trials. Hepatology 1999; 29: 62-67.

5. Nault, JC, Reyniès, AD, Villanueva, A. A hepatocellular carcinoma 5-gene score associated with survival of patients after liver resection. Gastroenterology 2013; 145: 176-187.

6. Zhou, XD, Tang, ZY, Yang, BH. Experience of 1000 patients who underwent hepatectomy for small hepatocellular carcinoma. Cancer 2001; 91: 1479-1486.

7. Camma, C, Cabibbo, G. Prognostic scores for hepatocellular carcinoma: none is the winner. Liver Int 2009; 29: 478-480.

8. Subramaniam, S, Kelley, RK, Venook, AP. A review of hepatocellular carcinoma (hcc) staging systems. Chin Clin Oncol 2013; 2: 33.

9. Tsai, SL. Biomarker for determining predisposition and/or prognosis of hepatocellular carcinoma. US20070117100 A1 Patent, 2007.

10. Hsu, IC, Metcalf, RA, Sun, T. Mutational hotspot in the p53 gene in human hepatocellular carcinomas. Nature 1991; 350: 427-428.

11. Li, M, Zhao, H, Zhang, X. Inactivating mutations of the chromatin remodeling gene ARID2 in hepatocellular carcinoma. Nat Genet 2011; 43: 828-829.

12. Tomczak, K, Czerwińska, P, Wiznerowicz, M. The Cancer Genome Atlas (TCGA): an immeasurable source of knowledge. Contemp Oncol 2015; 19: A68-A77.

13. Li, B, Dewey, CN, Li, B. RSEM: accurate transcript quantification from RNA-Seq data with or without a reference genome. BMC Bioinformatics 2011; 12: 323.

14. Wilkerson, MD, Hayes, DN. ConsensusClusterPlus: a class discovery tool with confidence assessments and item tracking. Bioinformatics 2010; 26: 1572-1573.

15. Diboun, I, Wernisch, L, Orengo, CA. Microarray analysis after RNA amplification can detect pronounced differences in gene expression using limma. BMC Genomics 2006; 7: 252. 
16. Ritchie, ME, Phipson, B, Wu, D. Limma powers differential expression analyses for RNA-sequencing and microarray studies. Nucleic Acids Res 2015; 43: e47.

17. Rashedi, E, Mirzaei, A. A novel multi-clustering method for hierarchical clusterings based on boosting. In: 19th Iranian conference on electrical engineering (ICEE), Tehran, Iran, 17-19 May 2011, pp.1-4. New York: IEEE.

18. May, WL. Kaplan-Meier survival analysis. Berlin: Springer, 2009.

19. O'Quigley, J, Moreau, T. Cox's regression model: computing a goodness of fit statistic. Comput Methods Programs Biomed 1986; 22: 253-256.

20. Carter, RL, Morris, R, Blashfield, RK. On the partitioning of squared Euclidean distance and its applications in cluster analysis. Psychometrika 1989; 54: 9-23.

21. Subramanian, A, Kuehn, H, Gould, J. GSEA-P: a desktop application for gene set enrichment analysis. Bioinformatics 2007; 23: 3251-3253.

22. Lee HJ, Finkelstein D, Li X, et al. Identification of trans-membrane protein 88 (TMEM88) as a dishevelledbinding protein. J Biol Chem 2010; 285: 41549-41556.

23. J. Palpant, L. Pabon, J.S. Rabinowitz, B.K. Hadland, C.L. Stoick-Cooper, S. L. Paige, I.D. Bernstein, R.T. Moon, C.E. Murry, Transmembrane protein 88: a Wnt regulatory protein that specifies cardiomyocyte development, Development 140 (2013) 3799-3808.

24. Zhao, F. Lu, S. Cui, X. Zhang, W. Wang, E. Si, Z. Yuan, TMEM88 inhibits extracellular matrix expression in keloid fibroblasts, Biomed. Pharmacother. 95 (2017) 1436-1440.

25. Xu, L.X. Pan, Y.X. Ge, P. Li, X.M. Meng, C. Huang, J. Li, TMEM88 mediates inflammatory cytokines secretion by regulating JNK/P38 and canonical Wnt/ $\beta$-catenin signaling pathway in LX-2 cells, Inflammopharmacology 26 (2018) 1339-1348.

26. P. Cai, X.Y. Cheng, P.J. Chen, X.Y. Pan, T. Xu, C. Huang, X.M. Meng, J. Li, Transmembrane protein 88 attenuates liver fibrosis by promoting apoptosis and reversion of activated hepatic stellate cells, Mol. Immunol. 80 (2016) 58-67.

27. X. Ge, C.H. Wang, F.Y. Hu, L.X. Pan, J. Min, K.Y. Niu, L. Zhang, J. Li, T. Xu, New advances of TMEM88 in cancer initiation and progression, with special emphasis on Wnt signaling pathway, J. Cell. Physiol. 233 (2018) 79-87.

28. Zhang, X. Yu, G. Jiang, Y. Miao, L. Wang, Y. Zhang, Y. Liu, C. Fan, X. Lin, Q. Dong, Q. Han, H. Zhao, Y. Han, X. Han, X. Rong, S. Ding, E. Wang, E. Wang, Cytosolic TMEM88 promotes invasion and metastasis in lung cancer cells by binding DVLS, Canc. Res. 75 (2015) 4527-4537.

29. Yu, X. Zhang, Y. Zhang, G. Jiang, X. Mao, F. Jin, Cytosolic TMEM88 promotes triple-negative breast cancer by interacting with Dvl, Oncotarget 6 (2015) 25034-25045.

30. Ma, N. Feng, X. Yu, H. Lin, X. Zhang, O. Shi, H. Zhang, S. Zhang, L. Li, M. Zheng, M. Gao, H. Yu, B. Qian, Promoter methylation of Wnt/ $\beta$-catenin signal inhibitor TMEM88 is associated with unfavorable prognosis 
of non-small cell lung cancer, Cancer Biol Med 14 (2017) 377-386.

31. Li T, Fan J, Wang B, Traugh N, Chen Q, Liu JS, Li B, Liu XS. TIMER: A Web Server for Comprehensive Analysis of Tumor-Infiltrating Immune Cells. Cancer Res. 2017; 77:e108-10.

32. Lánczky A, Nagy Á, Bottai G, Munkácsy G, Szabó A, Santarpia L, Győrffy B. miRpower: a web-tool to validate survival-associated miRNAs utilizing expression data from 2178 breast cancer patients. Breast Cancer Res Treat. 2016; 160:439-46.

33. Li B, Severson E, Pignon JC, Zhao H, Li T, Novak J, Jiang P, Shen H, Aster JC, Rodig S, Signoretti S, Liu JS, Liu XS. Comprehensive analyses of tumor immunity: implications for cancer immunotherapy. Genome Biol. 2016; 17:174.

34. Danaher P, Warren S, Dennis L, D'Amico L, White A, Disis ML, Geller MA, Odunsi K, Beechem J, Fling SP. Gene expression markers of Tumor Infiltrating Leukocytes. J Immunother Cancer. 2017; 5:18.

35. Sousa S, Määttä J. The role of tumour-associated macrophages in bone metastasis. J Bone Oncol. 2016; 5:135-38.

36. Tang Z, Li C, Kang B, Gao G, Li C, Zhang Z. GEPIA: a web server for cancer and normal gene expression profiling and interactive analyses. Nucleic Acids Res. 2017; 45:W98-102.

37. Andrew L, Yang Gu, Pengpeng Liu, et al. TMEM88 is a prognostic biomarker and correlates with immune infiltrates in hepatocellular carcinoma. 2021 Nov 02. Preprint available at Research Square https://doi.org/10.21203/rs.3.rs-1024755/v1.

Figures
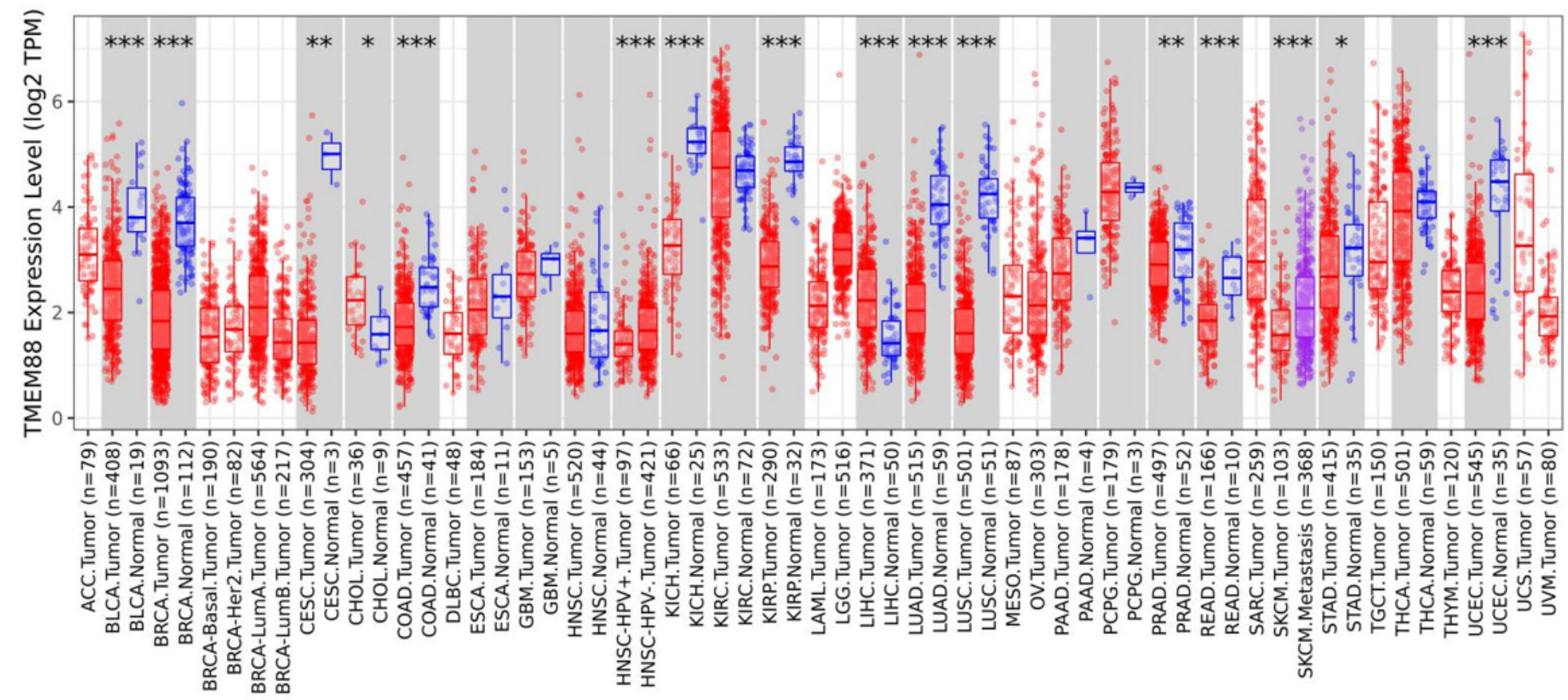
The level of TMEM88 expression in different tumor types from the TCGA database in TIMER. Note: ${ }^{\mathrm{P}}<0.05$, $\star * P<0.01, * \star * P<0.001$.

A

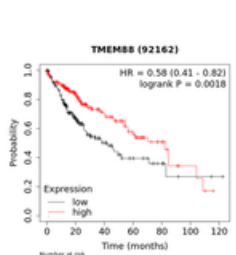

B $\mathrm{HCC}, \mathrm{PFS}$

C $\mathrm{HCC}, \mathrm{RFS}$

D $\mathrm{HCC}$, DSS
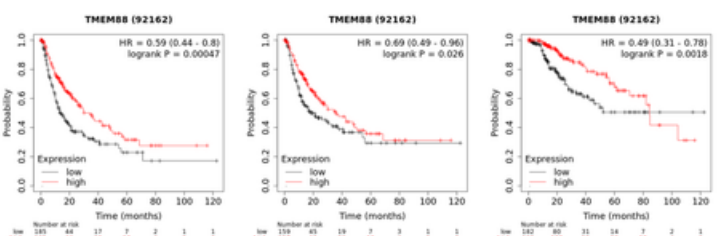

E Breast Cancer, OS

F Breast Cancer, PPS

G Breast Cancer, RFS

H Breast Cancer, DMFS
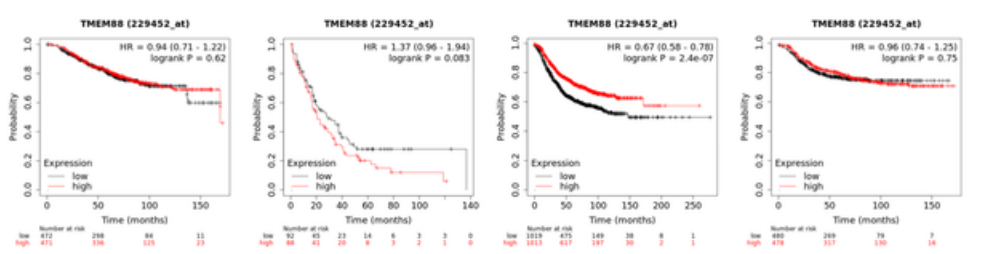

I Gastric Cancer, OS

Gastric Cancer,PPS

K Gastric Cancer ,FP

L Pancreatic Ductal
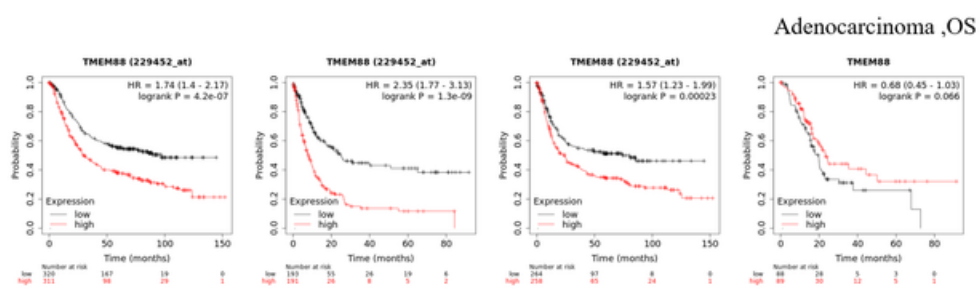

M Pancreatic Ductal

N Lung Cancer, os

O Lung Cancer, PPS

P Lung Cancer, FP

Adenocarcinoma ,RFS
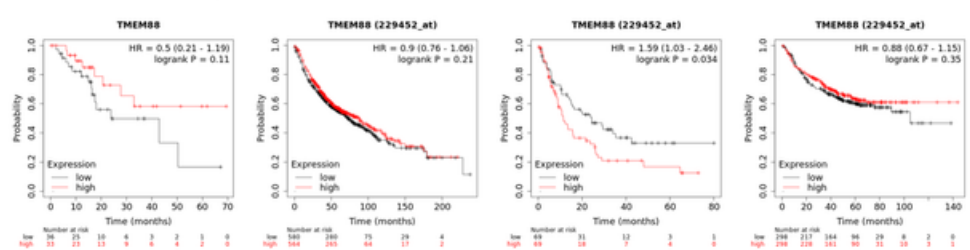

Q Ovarian Cancer, OS $\mathbf{R}$ Ovarian Cancer,PPS

S Ovarian Cancer, PFS
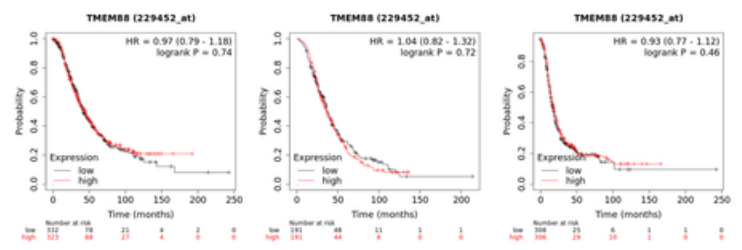

Figure 2

Kaplan-Meier survival curve analysis of the prognostic significance of high and low expression of TMEM88 in different types of human cancers using the Kaplan-Meier plotter database (A-S). (A-D) High TMEM88 expression was correlated with better OS, PFS, RFS and DSS in HCC cohorts ( $n=364, n=366, n=313, n=357)$. $(E-H)$ Survival curves of OS, PPS, RFS and DMFS in the breast cancer cohort $(n=1,879, n=458, n=4,929$, 
$\mathrm{n}=2,765)$. (I-K) OS, PPS and FP survival curves of gastric cancer. High TMEM88 expression was correlated with poor OS, PPS and FP $(n=875, n=498, n=640)$. $(L, M)$ OS and RFS survival curves of pancreatic ductal adenocarcinoma. High TMEM88 expression was correlated with better OS and RFS. $(n=177, n=69)$. (N-P) OS, PPS and FP survival curves of lung cancer. High TMEM88 expression was correlated with poor OS ( $\mathrm{n}=$ $1,925, n=344, n=982)$. (Q-S) OS, PPS and PFS survival curves of ovarian cancer $(n=1,656, n=782, n=1,435)$. OS: overall survival; PFS: progression-free survival; RFS: relapse-free survival; DSS: disease-specific survival; DMFS: distant metastasis-free survival; PPS: post progression survival; FP: first progression.

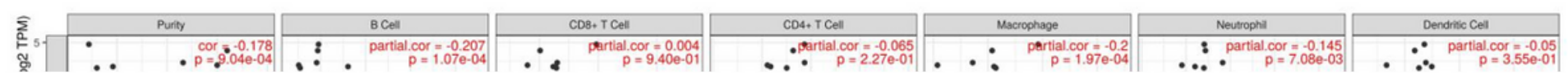

\section{Figure 3}

Correlation analysis of TMEM88 expression and infiltration levels of immune cells in HCC tissues using the TIMER database. TMEM88 expression in HCC tissues positively correlates with tumor purity and infiltration levels of CD8+ T cells, but negatively correlates with tumor purity and infiltration levels of B cells, CD4+ T cells, macrophages, neutrophils, and dendritic cells $(n=371)$.

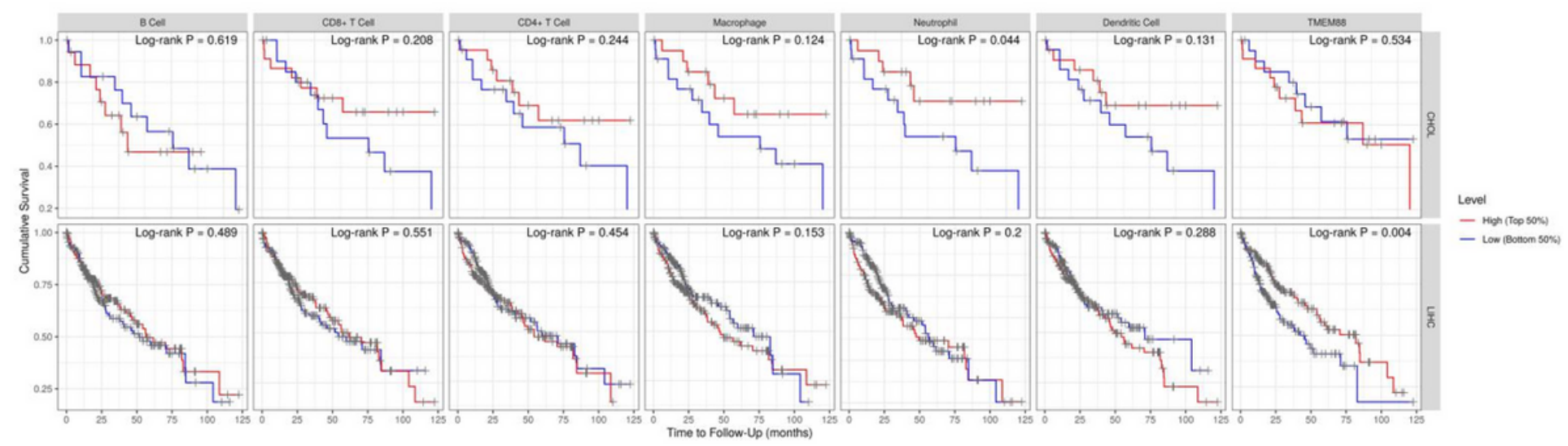

Figure 4

TIMER survival curve analysis of TMEM88 and other marker genes of immune cells in HCC ( $n=362,127$ dying) and $\mathrm{CHOL}$ ( $n=36,18$ dying) tissues using the TIMER database. 